\title{
Le débat public comme agir territorial : méthodes qualitatives
}

Julien Angelini

\section{OpenEdition}

1 Journals

Édition électronique

URL : http://journals.openedition.org/communicationorganisation/1482

DOI : 10.4000/communicationorganisation. 1482

ISSN : 1775-3546

Éditeur

Presses universitaires de Bordeaux

Édition imprimée

Date de publication : 1 décembre 2010

Pagination : 171-180

ISBN : 978-2-86781-743-4

ISSN : 1168-5549

\section{Référence électronique}

Julien Angelini, «Le débat public comme agir territorial : méthodes qualitatives », Communication et organisation [En ligne], 38 | 2010, mis en ligne le 01 décembre 2013, consulté le 19 avril 2019. URL :

http://journals.openedition.org/communicationorganisation/1482 ; DOI : 10.4000/

communicationorganisation. 1482 


\title{
Le débat public comme agir territorial : méthodes qualitatives.
}

\author{
Julien ANGELIN|or
}

Se positionner scientifiquement en intelligence territoriale revient à étudier la construction du territoire par les acteurs qui le composent, et ce, à travers des processus informationnels et communicationnels. Cet objet « territoire » est par nature complexe, et sa délimitation tant spatiale que sémantique reste problématique. La dimension anthropologique de l'intelligence territoriale aborde la capacité collective que nous développons pour co-construire du sens dans nos espaces de vie, dans notre rapport à la Terre. Ainsi, c'est le rapport entre la communauté territoriale, la médiation des représentations et la structuration du vivre-ensemble qui est un sujet central dans notre approche. A travers un projet territorial particulier, nous avons mis à l'épreuve une méthodologie qualitative et compréhensive pour aborder notre terrain sous le prisme de l'intelligence territoriale.

\section{Systémique, complexité et constructivisme}

Notre approche de l'intelligence territoriale adopte une vision systémique du territoire et considère la complexité du construit social engagé par ses acteurs. Penser les choses comme étant organisées en système revient immédiatement à déceler la structure des phénomènes considérés. La question de la forme apparaît alors puisqu'il s'agira dans le réel de faire émerger le tout constituant en identifiant ses éléments, leurs liens et les replacer dans leur contexte d'évolution. Le système appelle donc inévitablement au cadrage du projet territorial. Celui-ci nous apparaît comme relevant d'une nature complexe. Le point de départ est donc le suivant : accepter a priori que le phénomène que nous observons ne saurait être réduit à un modèle simplifiant. Mais c'est aussi accepter que les nécessaires cadrages de l'observation puissent nous rendre partiellement aveugles (E. Morin, 2005, p. 19). Là où nous tentons de limiter

01. Julien Angelini est docteur en Sciences de l'information et de la communication, Ater Sic à l'Université de Corse et membre de I'UMR CNRS 6240 LISA. Sa thématique de recherche porte sur le concept d'intelligence territoriale. Sa thèse est intitulée : "De la complexité de l'intégration des acteurs dans le développement local : approche par l'intelligence territoriale. Le cas du projet d'extension portuaire de Bastia » 
les mutilations, disjonctions et simplifications, subsistent cependant certaines dimensions que notre regard ne peut totalement appréhender. Pour ne pas nous écarter d'une démarche scientifique, qui au-delà de l'observation doit nécessairement proposer une explication, nous nous référons au paradigme de la complexité tel qu'il est présenté par Edgar Morin.

\section{L'intelligence territoriale}

Le territoire est donc à appréhender également dans le registre du complexe. Tangible, nous l'aménageons, asseyant ainsi nos modes de développement. Les évolutions technologiques nous permettent désormais de projeter le terrain dans l'immatériel cyberespace, rendant complexe et subtile l'articulation du quotidien entre les sphères matérielles et immatérielles. Le territoire est également symbolique et inscrit comme une représentation dans la mémoire collective de ses acteurs. Le partage des représentations du territoire s'appuie autant sur des bases communément admises, que sur les divergences que la discussion fait émerger. En cela le territoire est un projet, dans la mesure où il est incertain, indéterminé et en perpétuelle construction. Cette construction est l'œuvre collective des acteurs partageant un espace de vie, et ainsi engagés dans la médiation des représentations du territoire. Nous pensons que cette capacité collective à entreprendre la projection locale du développement, place le territoire comme échelle de référence pour une gouvernance renouvelée. Il serait ainsi le point de référence à partir duquel peut se mettre en mouvement la subsidiarité active (P. Calame, 2003). L'articulation des échelles de décisions, nécessaire au développement local, doit se baser sur une logique ascendante de la récolte d'informations. La production collective de l'ensemble des acteurs du territoire doit être la source d'information référentielle pour l'action décisionnelle.

En considérant l'importance première du lien social, nous acceptons ainsi le postulat suivant : appréhender scientifiquement un phénomène social relevant de l'organisation de la vie en collectivité nécessite une approche descriptive et analytique en termes d'information et de communication. Nous faisons le choix de recentrer l'intelligence territoriale en tant que processus informationnel et communicationnel, émergent et ascendant. Elle sera par essence protéiforme car elle est une émanation humaine, sociale et culturelle ; elle est donc complexe, et en évolution permanente. De manière synthétique nous pouvons proposer que l'intelligence territoriale étudie les processus d'émergence de l'intelligence sociale et citoyenne dans le traitement des problématiques de gestion du territoire.

\section{Projet de territoire et débat public}

Notre observation s'articule autour d'un questionnement relatif au partage des représentations du développement territorial. L'aménagement du territoire est en France historiquement conçu dans une vision descendante, c'est- 
à-dire correspondant à des décisions élaborées par les pouvoirs publics sur la base de l'expertise technique, et appliquées dans une perspective descendante. Ce processus correspond au modèle de démocratie représentative et délibérative structurant les échelles de décision du niveau national au niveau local. Cette posture implique l'existence d'un système de communication de masse, destiné à asseoir la légitimité des actions entreprises par l'exécutif territorial. Le problème se pose quand la population ou du moins une partie de la société civile est résistante au changement. Dès lors, la logique descendante est bousculée et ainsi, survient un questionnement s'articulant autour du modèle de développement.

Dans le cas du projet d'extension du port de Bastia, nous nous trouvons confrontés à ce type de problématique. La réaction de certains acteurs du territoire a créé un système communicationnel mettant au premier plan la question des représentations du développement et de l'appropriation du projet territorial. La situation d'attente quant à une décision formelle de la Collectivité Territoriale de Corse dans ce dossier a laissé la place à une circulation d'information dans l'espace public mettant en jeu l'évolution des représentations de l'essence même du projet.

Nous considérons l'importance de la mise en place du premier débat public organisé en Corse par la CNDP ${ }^{02}$. Le nombre d'acteurs décisionnaires concernés et engagés dans la délibération première de la Collectivité Territoriale de Corse est important. Le nombre et l'importance des institutions concernées nécessitent déjà en soi une approche sous l'angle de l'intelligence territoriale. Mais la tenue d'un débat public, ouvert à l'ensemble des citoyens désireux de prendre part aux discussions, nous a semblé représenter un cas particulièrement intéressant à aborder. Une première étude des retombées médiatiques du débat public nous permet de constater deux choses qui retiennent notre attention. Le débat public semble faire l'objet d'une vive opposition entre des acteurs tenant des positions très contradictoires. Par ailleurs, à la lecture du bilan du débat public, il semble qu'aucun consensus solide n'apparaisse entre les parties prenantes, laissant présager une délibération compliquée au niveau de l'assemblée de Corse.

Ainsi, il nous apparaît que l'ouverture à la participation citoyenne dans le processus décisionnel concernant l'évolution du port de commerce de Bastia est bien un phénomène observable, du point du vue de la production et de la circulation de l'information, au sujet du développement territorial. Nous notons à ce sujet une caractéristique fondamentale de ce phénomène. Il s'agit du caractère obligatoire de la tenue d'un débat public nécessairement cadré par la CNDP. Voilà le point qui constitue l'articulation de notre questionnement. Nous soulignons pour l'intelligence territoriale, le caractère primordial de la mise en œuvre de processus ascendants concernant la participation des

02. Commission Nationale du Débat Public 
individus composant les territoires en développement ou en mutation. Le cas du port de commerce de Bastia semble présenter les caractéristiques d'une situation idoine pour éprouver notre approche théorique. Il s'agit de l'évolution ou de la mutation d'un équipement structurant venant modifier un territoire dans sa morphologie et sa dynamique. De plus, la portée économique de l'outil dépasse le cadre de la localité bastiaise, ce qui accroît la complexité du rapport entre l'impact local et l'intérêt régional. Ainsi, nous pouvons formuler une première interrogation en ces termes : comment se serait structurée la communication concernant le plan de développement du port de Bastia dans l'espace public en l'absence de l'intervention de la CNDP ? En effet, le caractère obligatoire de l'intervention de la CNDP donne probablement une forme particulière à la production de l'information et son échange entre les acteurs. Notre centre d'intérêt se situe donc le rapport entre débat public et espace public. Il nous faut étudier et cerner les contraintes et enjeux de l'organisation d'un débat public pour dégager les incidences de ce dernier sur l'animation de l'espace public. Nous pourrions présenter la problématique de la formalisation de notre terrain de recherche de la manière suivante : la CNDP à travers l'organisation des débats publics vient-elle se substituer à un espace public désormais défaillant?

Notre interrogation vient se placer en résonance de l'importance que nous accordons à l'existence et à la vitalité d'un espace public pour le développement territorial. Pour que notre interrogation ne s'engage pas dans un sens unique de par sa forme tautologique, nous l'affinerons en formulant notre hypothèse générale ainsi : le débat public est il une instance d'émergence de l'intelligence territoriale?

Ainsi, nous acceptons le fait que la période pendant laquelle se déroulent les réunions publiques pour le développement du port de Bastia, constitue une occupation importante de l'espace public, en termes de production et d'échange d'informations. Cependant, il est fort probable que l'espace public ne se résume pas au seul débat public.

\section{Méthodologie qualitative}

Nous avons élaboré une méthodologie de recherche qualitative et factuelle, afin de nous positionner dans une optique compréhensive de la problématique abordée. La référence à la méthodologie développée par Edgar Morin dans le cadre de l'étude sur la rumeur d'Orléans (E. Morin, 1982), constitue selon nous une base de réflexion adéquate pour atteindre notre objectif. Nous cherchons moins ici à démontrer qu'à montrer. En nous attachant à des traces, à des faits tangibles que nous situerons dans un contexte de succession, nous donnons la priorité à des éléments factuels qui pourront caractériser l'inscription matérielle des représentations du territoire. En constatant que débat public n'a pas permis de faire émerger un consensus autour d'un projet, et sur la base de notre connaissance du terrain, nous découvrons les 
chemins de l'information dans l'espace public du système territorial. Nous constituons un corpus qui est une collecte de tous les supports d'information et de communication que nous jugeons comme participant de la ressource informationnelle territoriale ${ }^{03}$. Cela, afin de porter un regard médiologique sur la matérialisation des stratégies de communication engagées par les parties prenantes du débat public, et plus largement par tous ceux qui produisent de l'information au sujet du développement du port de Bastia.

Le travail de terrain a connu trois phases distinctes. Il a commencé par un recueil de données essentiellement basé sur une veille Web. Il s'agissait de récupérer des informations permettant de confirmer une première approche informelle de la problématique. Cela a permis de prendre connaissance du caractère polémique du débat public et de travailler plus en profondeur cet aspect du terrain. Ne possédant au départ que le dossier du débat, nous nous sommes mis en quête de l'ensemble des documents officiels publiés dans le cadre du débat par le maître d'ouvrage. Ce travail de collecte, pour être le plus complet possible, a occasionné des rencontres au cours desquelles nous avons pu récupérer un ensemble d'informations confirmant que le débat public n’avait pas laissé ses participants indifférents. Cette première phase a précisé les hypothèses appliquées au terrain et dirigé vers la méthode d'enquête proposée.

Une deuxième phase était engagée. La recherche documentaire s'élargit alors à l'ensemble des supports communicationnels concernant le développement du port de Bastia. Il s'agissait de procéder à une collecte, si possible exhaustive, des traces laissées dans le registre de l'information et la communication. Nous nous sommes concentré initialement sur une veille web et médiatique afin de repérer l'évolution des publications et les possibles échanges entre les acteurs du débat. L'étude des parutions médiatiques me servant alors à définir les acteurs que je devrais rencontrer sur le terrain. La veille informationnelle s'est établie de la sorte :

- Consultation des archives de la presse écrite.

- Consultation des archives radiophoniques.

- Consultation des archives télévisuelles.

- Mise en place d'outils de veille automatique sur le Web.

- Recherche d'événements particuliers (manifestations, réunions publiques, distribution de tracts, affichages, tags...).

Une attention particulière étant portée au web pour sa capacité à diffuser des informations émanant de toute initiative individuelle et citoyenne. Cette

03. "La ressource informationnelle territoriale préexiste au collectif puisque chaque individu en porte les ferments. Elle émerge et apparaît dans la construction partagée de formes communes. Elle se situe dans une évolution récursive car elle est le produit du collectif qu'elle produit en retour. Elle conditionne les frontières poreuses (P. Dumas, 2006) de la territorialité en émergeant du territoire signifiant. Constamment disponible, elle est le ciment et le résultat d'une forme d'intelligence partagée que nous pourrions nommer l'intelligence collective (P. Levy, 1997) » (J. Angelini, 2007, p. 10). 
deuxième phase précédait l'enquête de terrain. Considérant que nous avions une connaissance suffisante de l'état des lieux relatif au projet, nous pouvions dès lors procéder à une enquête de terrain basée sur l'enchaînement des rencontres se précisant continuellement dans la progression de l'investigation. La tenue d'un journal a durant cette phase permis de consigner les éléments recueillis au travers des rencontres. Le recueil de données a amené à prendre la décision d'enregistrer certains entretiens, en raison de leur pertinence et de la volonté des individus de répondre concrètement à nos questions.

\section{Esthétique de la communication et agir territorial}

Nous n'observons pas, directement dans ce cas là, le développement d'une intelligence communicationnelle, dans le sens d'une adhésion à un socle de valeurs communes, permettant la co-écriture du projet territorial dans une instance volontairement partagée. L'action conjointe des parties prenantes produit de l'information réduisant l'incertitude (E. Morin, 1990) et réduisant les zones d'ombre ou de secret. Cela favorise la circulation sans entraves de l'information, caractéristique nécessaire d'une société de l'information sans embargo (N. Wiener, 1962). Ce recul de l'entropie est proportionnel au degré d'ordre dans le système (N. Wiener, 1962). Même quand les informations échangées sont contradictoires, elles sont structurées dans une forme de temporalité partagée, participant d'une mise en scène médiatique conforme à la volonté de permettre la bonne publicité (J. Habermas, 1987) des propos. Ainsi, le triangle - porteur de projet, collectif opposant, population - participe de cette circulation permettant de penser que le message a bien voyagé dans l'océan du bruit (D. Bougnoux, 2001). Le maintien de cette systémique de la communication nous montre que le lien et la présence du sujet semblent primer sur la validité technique du contenu. La capacité des parties prenantes à transférer les indices (C.S. Pierce, 1978) d'une sensibilité à partager, c'està-dire une issue au-delà des mots (D. Bougnoux, 2001), marque la singularisation d'un type de communication qui peut marquer l'opinion en accédant à la matérialisation d'un signe culturel (R. Debray, 1991). Le récepteur a la capacité de trouver dans un échange médiatique une interprétation du code, lui offrant la capacité de replacer le message dans une perspective symbolique et de l'associer à un socle de valeurs. C'est cela qui donne sa force au primat de la relation (D. Bougnoux, 1993). Notre terrain nous a montré que l'agir stratégique avait encore une pertinence pour décrypter les stratégies de communication institutionnelle. Il nous a aussi montré que le développement d'un agir communicationnel pouvait le remettre en cause et prendre de la consistance en investissant la sphère médiatique (J. Habermas, 1987). Le prisme systémique nous permet de penser que l'avenir du territoire se trouve dans cette activité complexe, pouvant relever d'une certaine esthétique de la communication que nous pourrions dénommer l'agir territorial. Il s'agirait du construit complexe de l'activité systémique des acteurs du territoire. Ce 
dernier se structurant à travers les formes contradictoires de la médiation collective. L'agir territorial serait le processus d'assimilation et d'accommodation (J. Piaget, 1966) du territoire, signe de la construction de son intelligence.

\section{Conclusion}

Notre travail s'achève sans que nous ayons pu observer la décision de la Collectivité Territoriale de Corse sur la suite à donner au projet. De nouvelles études d'impact, notamment écologiques, ont été ordonnées. Si les porteurs du projet et la nouvelle majorité territoriale affichent leur position pour un nouveau port de commerce, les opposants semblent ne pas avoir dit leur dernier mot. Nous pensons donc que le débat public participe de l'émergence d'une forme d'intelligence dans la mesure où la production d'informations contradictoires constitue la propension du système à s'organiser entre ordre et désordre (E. Morin, 2005). Mais nous pensons qu'il ne serait pas pertinent d'éluder la circulation implicite ou informelle de l'information. D'une part, il faut à notre sens prêter une attention particulière aux effets médiologiques que nous avons évoqués. Beaucoup d'effets indirects comme, les effets médiologiques, participent de la détermination des positions sur l'opportunité d'un projet. D'autre part, la circulation à travers les discussions dans les lieux publics, de la confrontation des avis, est une part importante qui n'est jamais accessible de manière matérielle. Nous proposerons donc de considérer la situation, ici mise en lumière comme une forme d'agir territorial, correspondant à une mise en synergie imparfaite des intelligences du territoire. Nous postulons de la nécessité de faire appel à toutes les connaissances, toutes les intelligences pour élaborer le projet local. Notre étude nous montre cependant qu'il s'agit d'une forme d'idéal difficilement accessible. Ainsi, nous avons mis en évidence ce dont le territoire est capable, dans la situation-problème observée. A notre sens, l'intelligence territoriale est en marche et la contradiction observée doit être analysée comme le signe du fonctionnement de la démocratie. La mise en œuvre de l'intelligence territoriale ne peut être l'œuvre unilatérale d'une catégorie d'acteur. Elle émerge des volontés de participation à l'élaboration du projet territorial. Elle représente plus une forme de pensée commune et complexe, qu'une décision délibérative. La conclusion rédigée par le Président de la Commission Particulière du Débat Public sur le projet d'extension du port de Nice commence par la phrase suivante : "On ne sort jamais indemne d'un grand débat public ». Le débat territorial peut laisser des cicatrices dans les mémoires, cristalliser de profonds antagonismes et paralyser temporairement les décideurs. L'évolution culturelle du territoire réside peut-être dans sa capacité à s'engager dans une dynamique ascendante de la définition du développement local. Nous savons cependant que les projets d'envergure, structurant durablement le territoire, sont le plus souvent l'émanation d'une instance institutionnelle mettant en ouvre le développement sur la base d'un constat d'ordre technique ou scientifique. Si ces projets restent de la compé- 
tence des instances de gouvernement, il demeure néanmoins nécessaire que les acteurs territoriaux mobilisent à travers des processus d'information et de communication, les intelligences diverses du territoire.

\section{BIBLIOGRAPHI}

ARENDT Hannah, 1972, la crise de la culture, Gallimard, collection Folio, Paris, 1972. $380 \mathrm{p}$.

ATTALI Jacques, 2006, Une brève histoire de l'avenir, Fayard, Paris, 2006, 422 p.

BOUGNOUX Daniel, Sciences de l'Information et de la Communication, Larousse, 1993, Paris, 808 p.

BOUGNOUX Daniel, Introduction aux Sciences de la Communication, La Découverte, 2001, Paris, 125 p.

BRESSON GILLET Sylvie, 2010, L'enjeu communicationnel du débat public ITER en Provence, Les Enjeux de l'information et de la communication, http://w3.u-grenoble3. fr/les_enjeux.

CALAME Pierre, La démocratie en miettes, Pour une révolution de la gouvernance Charles Léopold Mayer Descartes et Cie, Paris, 2003, 331 p.

CROZIER Michel, FRIEDBERG Erhard, L'acteur et le système, Les contraintes de l'action collective, Seuil, collection sociologie politique, Paris, 1977, 436 p.

CROZIER Michel, TILLIETTE Bruno, La crise de l'intelligence, Essai sur l'impuissance des élites à se réformer, InterEditions, Paris, 1995, $200 \mathrm{p}$.

DEBRAY Régis, Le moment fraternité, Gallimard, Paris, 2009, 367 p.

DEBRAY Régis, Cours de médiologie générale, Gallimard, collection Bibliothèque des Idées, Paris, 1991, 395 p.

GIDDENS Anthony, La constitution de la société, Presses universitaires de France, Paris, 2005, $471 \mathrm{p}$.

HABERMAS Jürgen, Théorie de l'agir communicationnel, Tome 1, Rationalité de l'agir et rationalisation de la société, Fayard, collection L'espace du politique, Paris, 2005, 448 p.

HABERMAS Jürgen, Théorie de l'agir communicationnel, Tome 2, Pour une critique de la raison fonctionnaliste, Fayard, collection L'espace du politique, Paris, 2005, 477 p.

HERBAUX Philippe, Intelligence Territoriale, Repères théoriques, L'Harmattan, collection Questions contemporaines, Paris, 2007, 194 p.

LEVY Pierre, L'intelligence collective, Pour une anthropologie du cyberspace, La Découverte, Poche Essais, Paris, 1997, 245 p.

MATTELART Armand, L'Invention de la communication, La Découverte, Poche, Sciences humaines et sociales, Paris, 2007, 375 p.

MATTELART Armand \& Michèle, Histoire des théories de la communication, La Découverte, Collection Repères, $3^{\text {ème }}$ édition, Paris, 2004, 123 p.

MAUPERTUIS Marie-Antoinette (Sous la dir. de), La corse et le développement durable, Albiana, Ajaccio, 2010, $350 \mathrm{p}$.

MORIN Edgar, La rumeur d'Orléans, Points / Essais, Seuil, Paris, 1982, 252 p.

MORIN Edgar, Introduction à la pensée complexe, Points / Essais, Seuil, Paris, 2005, 158 p. 
MUCCHIELLI Alex (sous la dir. de), Dictionnaire des méthodes qualitatives en sciences bumaines 2009, Armand Colin, $3^{\text {ème }}$ édition, Paris, 2009, 296 p.

MUCCHIELLI Alex, Les sciences de l'information et de la communication, $4^{\text {ème }}$ Edition, Hachette Supérieur, collection Les fondamentaux Sciences humaines, Paris, 2006, 160 p.

PAILLART Isabelle \& ROMEYER Hélène, Les débats publics autour des questions de sciences et de techniques et leur dimension communicationnelle, Les Enjeux de l'information et de la communication, http://w3.u-grenoble3.fr/les_enjeux 2010

PELISSIER Maud \& PYBOURDIN Isabelle, L'intelligence territoriale. Entre structuration de réseau et dynamique de communication, $94 \mathrm{LCN} \mathrm{n}^{\circ} 4$, Intelligence économique, pp 93-109. 2009

RENAUT Alain, Qu'est-ce qu'une politique juste ?, essai sur la question du meilleur régime, Le Livre de Poche, collection biblio essais, Paris, 2005, 276 p.

ROSANVALLON Pierre, L'Etat en France de 1789 à nos jours, Seuil, collection Univers Historique, Paris, 1990, 369 p.

WATZLAWICK Paul \& al., Une logique de la communication, Seuil, Points, Paris, 1979, 280 p.

WEILL Agnès, Le débat public : entre médiation et mise en scène. Retour sur le débat public "gestion des déchets radioactifs, Les Enjeux de l'information et de la communication, http:// w3.u-grenoble3.fr/les_enjeux 2010

Résumé : L'objectif poursuivi dans cet article est de proposer au lecteur une analyse compréhensive de processus informationnels et communicationnels à travers une étude de cas originale. Dans le cadre d'un travail doctoral, l'auteur a abordé la question de l'extension portuaire de la ville de Bastia et plus particulièrement les phénomènes communicationnels liés à l'organisation d'un débat public - le premier en Corse - sous l'égide de la Commission Nationale du Débat Public. L'intérêt de l'étude réside dans l'observation des échanges dans l'espace public au-delà du cadre du débat public. Sous un angle systémique et adoptant la posture de l'observation participante, l'auteur analyse la construction et l'organisation du projet territorial.

Mots clés : territoire, système, développement local, méthodes qualitatives.

\begin{abstract}
The aim in this article is to provide the reader with a qualitative analysis of informational and communicational processes through an original case study. As part of a doctoral work, the author has addressed the issue of Bastia city port extension and especially communicational phenomena related to the organization of public debate - the first one in Corsica - managed by the National Commission of Public Debate.

The interest of the study is the observation of mediation in the public sphere beyond the context of public debate. Under a systemic perspective and adopting a participant-observer posture, the author discusses the construction and the organization of the territorial project.
\end{abstract}

Key word : territory, system, local development, qualitative methods. 